\title{
Eccentric activation and muscle damage: biomechanical and physiological considerations during downhill running
}

\author{
Roger G. Eston DPE, Jane Mickleborough MCSP MSc ${ }^{\dagger}$ and Vasilios Baltzopoulos PhD ${ }^{\dagger}$ \\ Division of Health and Human Performance, University of Wales, Bangor UK and ${ }^{\dagger}$ Department of Movement \\ Science and Physical Education, University of Liverpool, Liverpool, UK
}

\begin{abstract}
An eccentric muscle activation is the controlled lengthening of the muscle under tension. Functionally, most leg muscles work eccentrically for some part of a normal gait cycle, to support the weight of the body against gravity and to absorb shock. During downhill running the role of eccentric work of the 'anti-gravity' muscles - knee extensors, muscles of the anterior and posterior tibial compartments and hip extensors - is accentuated. The purpose of this paper is to review the relationship between eccentric muscle activation and muscle damage, particularly as it relates to running, and specifically, downhill running.
\end{abstract}

Keywords: downhill running, biomechanics, physiology, muscle damage

\section{Mechanisms of muscle injury}

Recent evidence indicates that skeletal muscle damage may be the primary mechanism contributing to muscle soreness and strength loss after eccentric exercise $^{1-5}$. However, the relationship between skeletal muscle damage, muscle soreness and loss of muscle force is unclear. Muscle soreness peaks well before muscle damage indices and muscle weakness appears to be at its worst immediately post-exercise. There is substantial evidence that eccentric contractions cause more damage than other types of contraction $^{1,2}$. Ultrastructural changes within skeletal muscle fibres were first demonstrated by Friden $e t$ al. $^{3}$ following an exercise protocol of repeated stair descents which caused severe delayed onset muscle soreness (DOMS). Post-exercise samples showed myofibrillar disturbances consisting of Z-band disruption and streaming.

Two hypotheses, metabolic overload and mechanical strain, have been suggested as causative mechanisms for muscle damage. As eccentric activation causes most muscle damage, it suggests that high local muscle tensions are in some way more important than extreme metabolic demand in the aetiology of DOMS injury ${ }^{6}$.

Address for correspondence: Dr R. G. Eston, Division of Health and Human Performance, Ffriddoedd Building, Victoria Drive, University of Wales, Bangor, LL57 2EN, UK
Appell et al. ${ }^{7}$ presented evidence to suggest that the mechanisms producing muscle damage after level endurance running and downhill running were not the same. A comparison was made of the histological structure of the rat soleus muscle, subjected either to a level endurance run or a downhill run. The loss of striation pattern seen in $15 \%$ of fibres at $48 \mathrm{~h}$ were predominantly in fibres which were glycogen depleted. This suggests a metabolic aetiology for the damage. Glycogen depletion was present in $25 \%$ of the fibres immediately after level running and increased to $33 \%, 48$ hours later. There were similar levels of lysosomes in the muscle fibres. This, combined with an immediate $16 \%$ loss of sarcomere organization, suggested an autophagic response, due to metabolic exhaustion and enzyme leakage. In contrast, however, the downhill running muscle had higher disruption levels ( $33 \%$ of fibres with immediate loss of sarcomere organization) but no glycogen depletion or lysosome occurrence in the fibres. This lack of glycogen depletion and absence of autophagic response supports a mechanical origin of muscle damage ${ }^{8}$.

\section{Strength loss}

Perhaps one of the more noticeable effects of eccentric exercise induced by downhill running is the acute loss of strength which continues for some days after the damaging exercise ${ }^{9-12}$. Short-term strength loss is also experienced after concentric and isometric exercise. The longer duration strength loss, associated with eccentric exercise, has been attributed to sarcoplasmic reticulum damage. This latter theory has been postulated by Clarkson et al. ${ }^{9}$ on the assumption that, if the sarcoplasmic reticulum is damaged, the restoration of normal calcium levels would be delayed and the ability to generate normal force levels would be impaired. Indeed, altered sarcoplasmic reticulum function and consequent intracellular regulation of free calcium concentration has been reported after prolonged ${ }^{13}$ and high intensity exercise ${ }^{14}$ in animals.

An additional explanation for the loss of strength after eccentric exercise has been put forward by Clarkson et $a l^{9}$. They proposed that the sarcomeres are stretched out by performance of the lengthening 
action. Sarcomeres are shorter towards the end of the muscle fibres ${ }^{15}$. If the lengthening action pulls some of the sarcomeres apart, the overlap between the actin and myosin filaments would be reduced which would reduce the number of cross bridges that could form. However, they acknowledged that there are as yet no data to substantiate such a theory.

In contrast to the rapid recuperation of strength after concentric exercise, the loss of strength after eccentric exercise is much longer lasting. The length of time the muscle requires to regain full strength does not appear to be established, as studies tend to measure only until around 10 days, where there is still usually a significant reduction of strength compared to pre-trial values. Eston et al. ${ }^{12}$ compared uphill and downhill running at similar exercise intensity $\left(80 \%\right.$ of the maximal heart rate, $\left.\% \mathrm{HR}_{\max }\right)$. They reported a decrease in the strength of the knee extensors from $270( \pm 40 \mathrm{~N} \mathrm{~m})$ to $115( \pm 34)$ immediately following five 8-min bouts of downhill running at $-10 \%$ interspersed with $2 \mathrm{~min}$ rest periods. Strength was still lower $(225 \pm 49 \mathrm{~N} \mathrm{~m})$ after 7 days. There was no change in strength after running uphill at a similar metabolic cost. Finney et al. ${ }^{16}$ using a similar protocol, measured concentric and eccentric isokinetic strength of the knee extensors. They reported similar strength losses for both concentric and eccentric strength, with values returning to normal after 4 days.

The degree to which strength decreases appears to be related to the relative length of the muscle during eccentric exercise. For example, Newham et al. ${ }^{17}$ observed that when the elbow flexors were exercised at long length, strength decreased to $75 \%$ of the initial values immediately after exercise and at $24 \mathrm{~h}$ later. Short length exercise produced much smaller changes in strength.

Muscle tenderness, which peaked at $48 \mathrm{~h}$, was also greatest in the muscles exercised at long length. Newham et al. ${ }^{17}$ therefore concluded that high force generation was not the only explanation for the undesirable consequences of eccentric exercise. It should also be noted that forces generated at long length are lower than forces generated at short length (100-250 N long, $150-375 \mathrm{~N}$ short $)^{17}$. Similarly, long length isometric maximal voluntary contractions (MVC) produce greater low-frequency fatigue and pain, with relatively lower force than for MVC at short length ${ }^{18}$.

Newham et al. ${ }^{17}$ therefore concluded that the differences in strength, after exercise at long and short length, were not just due to the magnitude of the force generated in the muscle. Changes in strength also appear to be related to the length at which the muscle is exercised. In downhill running the knee extensor muscle group is worked over a greater range than for uphill running, with more work at its longer length.

\section{Soreness and tenderness}

Tenderness, measured by pressure transducers (e.g. Penny and Giles Myometer, Christchurch, Dorset, UK) is greatest in the gluteus maximus, rectus femoris, vastus medialis, vastus laterali, tibialis anterior, gastrocnemius and biceps femoris when measured 2 days after downhill running ${ }^{12}$. The greatest tenderness occurred at the distal myotendinous junction of the muscles. This corresponds with previous investigations which have used different protocols of inducing damage ${ }^{19-21}$. The fact that this tenderness appears to be immediate ${ }^{19}$ rather than delayed may suggest that it originates in connective tissue. Pain experienced during active extension is accompanied by a decrease in resting angle ${ }^{19}$, which is perhaps due to tension on the damaged connective tissue.

\section{Creatine kinase}

Creatine kinase is found in skeletal muscle, cardiac muscle and the brain. Abnormally high circulating levels of enzymes such as creatine kinase are taken to reflect changes in the integrity of muscle fibre membranes - either damage to, or increased permeability, of the membrane to the enzyme. Eccentric activation of muscle has been widely implicated as the cause of large increases in plasma creatine kinase (PCK) after exercise ${ }^{17,22-24}$.

There appears to be a quantitive difference in the PCK response between high force, maximal eccentric exercise and downhill running. For example, after maximal eccentric exercise of the forearm flexors, Clarkson et al..$^{9}$ observed that PCK levels started to rise quickly after 2 days and peaked at 4 days at a level of about $2500 \mathrm{U} \cdot \mathrm{1}^{-1}$ High intensity eccentric exercise in untrained men produced a mean peak PCK response of $2143 \mathrm{u} \cdot 1^{-1}$ at 5 days ${ }^{25}$. Peak levels of around $1500-11000 \mathrm{u} \cdot \mathrm{l}^{-1}$ were reported by Newham et $a .^{26}$ after maximal eccentric exercise. After highintensity eccentric exercise of the knee extensors, PCK has also reached levels of $6988 \pm 1913 u \cdot l^{-1}$ at the third day after exercise ${ }^{27}$.

In contrast, PCK levels after downhill running seem to peak much earlier, at a much lower level. Examples of this are levels of $339 \pm 379.6 \mathrm{u} \cdot 1^{-1}$, observed by Byrnes et al. at $24 \mathrm{~h}^{28}$, and a peak at $24 \mathrm{~h}^{\prime}$ of between $425-460 \mathrm{u} \cdot 1^{-1}$ observed by Schwane et al. ${ }^{29,30}$. Studies from our laboratory showed peak levels of $400-580 \mathrm{u} \cdot 1^{-1}$ occurring 2 to 4 days after downhill running at $10 \%$ gradient ${ }^{12,16}$. PCK levels for downhill walking seem to reflect the relative intensity and duration of maximal eccentric exercise, with levels of between $700-1500 u \cdot l^{-1}$ between $4-7$ days $^{24}$.

There is a large difference in the working muscle mass of forearm flexors compared to the eccentrically working leg muscles in downhill running, which makes these contrasts even more striking. There is as yet no explanation for the difference in time course or magnitude of PCK levels between high force eccentric exercise and downhill running exercise ${ }^{9}$. Perhaps the reason for the disparity in magnitude is that high force eccentrics involve higher forces maintained through a longer strain range than downhill running.

\section{Oxygen drift during downhill running}

Despite its relative metabolic efficiency, a number of studies have shown a steady upward drift of $\dot{V} \mathrm{O}_{2}$ during downhill running 11, 28, 31-33. Sergeant and Dolan ${ }^{11}$ observed that oxygen uptake rose 
progressively from $45 \%$ of $\dot{V}_{\mathrm{O}_{2} \text { max }}$ after $10 \mathrm{~min}$ to $65 \%$ $\dot{V}_{2 \text { max }}$ at the end of a downhill running exercise. Pierrynowski et al. ${ }^{32}$ also observed an upward drift of $\dot{V} \mathrm{O}_{2}$ during downhill running and downhill training at a constant stride frequency, compared with a stable $\dot{V} \mathrm{O}_{2}$ during uphill training.

Iverson and McMahon ${ }^{34}$ found that the oxygen demand at a steady $3 \mathrm{~m} / \mathrm{sec}^{-1}$ reduced with increasing downhill gradient, until near $-0.10 \mathrm{rad}$, and then increased as the downhill angle grew steeper. At the angle of $-0.10 \mathrm{rad}$, the normal foot reaction force was found to be maximal. It is suggested that this gradient is the most efficient in terms of energy expenditure, making greater use of the stored elastic energy of the muscles, while causing most stress to the legs and feet.

Dick and Cavanagh ${ }^{31}$ compared the $\dot{V}_{2}$ of experienced runners, over two same-speed runs, 2 days apart, on the level and downhill. For the level run there were no differences in $\mathrm{O}_{2}$ consumption, integrated EMG (vastus lateralis and vastus medialis) or stride length after 10 and $40 \mathrm{~min}$. However, in the downhill run, there was a $10 \%$ increase in $\mathrm{O}_{2}$ consumption and a $23 \%$ increase in EMG activity in the same time period. It was hypothesized that the upward drift in $\dot{V}_{2}$ and EMG increase were a result of increased motor unit recruitment in the eccentrically activated muscles, caused by muscle/connective tissue damage and local muscle fatigue. However, Westerlind et al. ${ }^{33}$ have contradicted this argument. They observed increases in $\dot{V}_{2}$, heart rate and ventilation during two downhill runs at $40 \%$ peak $\dot{V} \mathrm{O}_{2}$. Upward drift of $\dot{V}_{\mathrm{O}_{2}}$ over time was similar for the two downhill runs $(15.6 \%$ and $14.7 \%$ respectively). Serum CK activity or perceived muscle soreness however was reduced during the second run. It was therefore suggested that the $\mathrm{VO}_{2}$ drift increase during downhill running is not related to muscle damage.

\section{Adaptation and training}

Previous training reduces the muscle soreness response and reduces morphological changes, performance changes and PCK activity in the blood $^{17,26,28,29,35-41}$. Soreness is reduced by training that involves eccentric contractions ${ }^{21}$ as concentric training does not reduce DOMS in subsequent eccentric exercise ${ }^{42}$. Similarly, in animals, the muscle damage that occurs during downhill running is prevented by downhill and level training, but not by uphill training ${ }^{36}$. The protective effect of a prior bout of exercise, that in itself may only produce minimal soreness, lasts for prolonged periods. Byrnes et al. ${ }^{28}$ observed that the muscle soreness response to downhill running was reduced by up to 6 weeks following an initial bout of downhill running. Similarly, Pierrynowski et al. ${ }^{32}$ found that as little as two 12 min bouts of downhill running at a gradient of $10 \%$ were sufficient to protect against the occurrence of DOMS in a subsequent downhill run 3 days later, which had produced DOMS in the control group. However, they also noticed that this training was not sufficient to prevent a 2-3 day reduction in muscular strength, suggesting that strength loss and DOMS may have different physiological origins.
We have examined the effect of a prior bout of maximal isokinetic eccentric exercise on DOMS, strength loss and serum $\mathrm{CK}^{16}$ following a downhill run in 10 male students. The experimental group performed 100 maximal, isokinetic eccentric activations at $0.52 \mathrm{rad} \cdot \mathrm{s}^{-1}$. Two weeks later the downhill run was performed on a motor driven treadmill. This consisted of five bouts of $8 \mathrm{~min}$ at a gradient of $-10 \%$ at a speed corresponding to $80 \%$ of the maximum heart rate. The control group performed the downhill run as above but without the prior isokinetic session. After isokinetic exercise, peak torque decreased by $10 \%(358.6 \pm 38.8$ to $323.0 \pm 26.9 \mathrm{~N} \mathrm{~m})$ immediately post-exercise and returned to baseline levels by day 4 . Serum creatine kinase activity increased by $403 \%$ $\left(86 \pm 22\right.$ against $\left.345 \pm 234 \mathrm{u} \cdot 1^{-1}\right)$ and rating of perceived soreness (RPS) increased from 0 to $4.8 \pm 2.8$ by day 4. Following the downhill run, post-exercise strength loss was lower in the trained group $(p<0.05)$ at both eccentric speeds $(7 \mathrm{~N}$ m against $68 \mathrm{~N} \mathrm{~m}$ at 0.52 $\mathrm{rad} \cdot \mathrm{s}^{-1}$ and $37 \mathrm{~N} \mathrm{~m}$ against $87 \mathrm{~N} \mathrm{~m}$ at $2.83 \mathrm{rad} \cdot \mathrm{s}^{-1}$ for the trained and untrained groups, respectively) and at the slower concentric speed $(22 \mathrm{~N}$ m against $64 \mathrm{~N} \mathrm{~m}$ for the trained and untrained group, respectively). There was less soreness and tenderness $(p<0.05)$ in the trained knee extensor muscle group. Strength also returned to baseline measures earlier for the trained group. Serum creatine kinase activity peaked on day 4 for the trained group $\left(319 \pm 109 u \cdot 1^{-1}\right)$ and on day 2 for the untrained group $\left(578 \pm 269 \mathrm{u} \cdot 1^{-1}\right)$ $(p<0.05)$. For the untrained group tenderness was greatest $(p<0.05)$ in the distal musculotendinous junction, whereas it was greatest in the muscle belly for the trained group. There were no significant differences in tenderness measurements for the gluteus maximus, anterior tibialis and gastrocnemius muscles between the two groups. The results suggest that a prior bout of isokinetic eccentric training reduces the severity of muscle damage, reduces the amount of strength loss and decreases the sensation of soreness and tenderness after downhill running.

Ebbeling and Clarkson ${ }^{43}$ suggested that the muscle repairs itself after the first bout of damage-inducing exercise and that adaptations within the muscle surrounding connective tissue were the reasons for the rapid training effect after one eccentric exercise bout. However, Clarkson et al. ${ }^{9}$ have now suggested that this theory is unlikely, as over time periods of 6 months, with the constant turnover of cellular components, the 'conditioned' fibres were unlikely to retain their adaptations. They hypothesized that the first bout of eccentric exercise adapts the motor-unit recruitment pattern over the movement range, to more equally distribute the force between the muscle fibres, reducing the chance of severe damage to any one motor unit. Motor skills are 'stored' for a long time, giving credence to this neurological theory; along with a cellular response as the reason for the long training effect.

\section{Biomechanics of downhill running}

During normal locomotion (walking and running) the extensor muscles in the lower limbs perform eccentric contraction during each stride to decelerate the centre 
of mass after the foot touches the ground ${ }^{44}$. Following downhill running, greater DOMS is produced in the gluteal muscles, the quadriceps, and the anterior and posterior tibial muscles, than for an equivalent bout of level running ${ }^{12}$.

Armstrong et al. ${ }^{2}$ suggested that it is primarily the eccentric contraction phase that causes muscle damage during normal level running since the highest tensions in the leg extensor muscles are produced whilst the muscles are lengthening after the foot touches the ground and the centre of mass is decelerating.

Few studies have examined the kinematic differences between downhill and level running and highlighted the differences in the knee angle between footstrike and peak flexion angle $e^{45,46}$. The overall change in angle is greater in downhill running, with peak flexion angle significantly greater. This means that the muscle is working eccentrically at a greater length during downhill running. As alluded to earlier, there appears to be a length-dependant component in the production of muscle damage ${ }^{17}$. In downhill running the knee extensor muscle group is worked over a greater length, with more work at its longer length. This is exemplified from the following kinematic data.

During downhill running, the overall change in knee angle from footstrike to peak flexion angle is much greater than in level running. Values cited in the literature for downhill and level running are $0.52 \mathrm{rad}\left(30.9^{\circ}\right)$ at a gradient of $-8.3 \%$ against $0.34 \mathrm{rad}$ $\left(19.3^{\circ}\right)^{45}, 0.61 \mathrm{rad}\left(35.1^{\circ}\right)$ at a gradient of $-10 \%$ against $0.47 \mathrm{rad}\left(27.2^{\circ}\right)^{46}$, and $0.57 \mathrm{rad}\left(32.5^{\circ}\right)$ at a gradient of $-9 \%$ against $0.36 \mathrm{rad}\left(20.5^{\circ}\right)$ (results from our laboratory). This movement takes place in the first half of the stance phase of gait, with peak flexion angle occurring significantly later $(40.7 \%$ and $33.6 \%$ of stance), for downhill and level running respective$1 y^{45}$. Values from our laboratory are $50.0 \%$ against $47.6 \%$ of the stance phase for downhill and level running respectively. This appears to be the major phase of negative or eccentric work for the knee extensors, which control knee flexion and absorb shock. The initial knee flexion angle at footstrike is less in downhill compared to level running $0.30 \mathrm{rad}$ $\left(17.0^{\circ}\right)$ against $0.43 \mathrm{rad}\left(24.6^{\circ}\right)$ and the peak flexion angle is greater $0.84 \mathrm{rad}\left(47.9^{\circ}\right)$ against $0.77 \mathrm{rad}$ $\left(43.9^{\circ}\right)^{45}$. Corresponding values for the initial knee flexion angle at heelstrike from our laboratory are $0.12 \mathrm{rad}\left(7.1^{\circ}\right)$ at $-9 \%$ gradient and $4 \mathrm{~m} \cdot \mathrm{s}^{-1}$, against $0.30 \mathrm{rad}\left(17.4^{\circ}\right)$ for the level running. Despite these differences the angle between leg and the vertical does not change during downhill running ${ }^{34}$.

In downhill running, the knee extensor muscle group is being actively strained to a greater degree than in level running, while undergoing a simultaneous contraction. Leiber and Fridén's ${ }^{47}$ work demonstrated that it is the degree of active strain on a contracting muscle, independent of the relative force exerted by the muscle, that produces the characteristic signs and symptoms of muscle damage, not found in level running.

The timing of maximum dorsiflexion is also later as a percentage of stance in downhill compared to level running (49.3\% against $45.0 \%$ ). Peak dorsiflexion velocity is the same for both downhill and level running, but there are significant differences in the timing, with peak values occurring later in stance $(28.6 \% \text { against } 21.4 \%)^{45}$.

However, there is no difference in the peak dorsiflexion angle at the ankle. This may explain why there is no greater incidence of muscle damage in the posterior calf muscles during downhill running. Most studies of downhill running and muscle damage have concentrated on the quadriceps group, with very little mention of posterior calf pain. The ankle is a less mobile joint than the knee, with dorsiflexion from neutral restricted to less than $0.44 \mathrm{rad}\left(25^{\circ}\right)^{48}$. This lack of range of movement may explain the finding of Winter ${ }^{49}$ that the ankle is a net generator of force, while the knee is a net absorber in level jogging. There may not be sufficient range in the ankle to let the calf muscles act efficiently as absorbers of force. Because of the functional position of the ankle, the posterior calf muscles may simply be more adapted to working at their long length, protecting them from muscle damage in downhill running. Power generation/absorption is the product of joint moment and joint angular velocity and is either a positive or negative quantity, depending on the directions of joint moment and angular velocity. Buczec and Cavanagh ${ }^{45}$ reported a much greater total of negative work in both knee and ankle in downhill, compared to level, running. Values for absorption at the knee were about twice those for the ankle in both downhill and level running. Specifically, the negative (absorption) work of the knee extensors in stance phase was $58 \mathrm{~J}$ and $30 \mathrm{~J}$ for downhill and level running, respectively. Negative work done on extensor muscles at the ankle was $26.1 \mathrm{~J}$ for downhill running compared to $12.6 \mathrm{~J}$ for level running. It was proposed that it was the higher power absorption requirements, rather than the greater range of joint movement in downhill running that caused the muscle damage and soreness. Overall in level running, it has been shown that the knee extensors absorb 3.5 times as much energy as they generate (69.2 J against 19.6 J), with the ankle plantarflexors generating 3 times more energy than they absorb (59.0 J against 19.6 J) ${ }^{49}$.

At the knee, the negative work period as a percentage of total stance time is significantly greater for downhill running (42.1\% against $35.7 \%$ ), with no significant difference in the duration of stance phase between downhill and level running. In the ankle the negative work period is also significantly greater for downhill running (43.6\% against $28.6 \%)^{45}$.

In the lower leg, the plantarflexors of the ankle soleus and gastrocnemius - work eccentrically to control the forward momentum of the leg over the foot in early stance phase and facilitate heel lift. The ankle extensors (dorsiflexors) tibialis anterior, extensor hallucis longus and extensor digitorum longus, also have a considerable eccentric work component in gait to control the descent of the forefoot ${ }^{48}$.

In a study of ground reaction forces during level and downhill running, support time was again almost identical between conditions ${ }^{50}$. Vertical impact peak force was $14 \%$ higher for downhill compared to level running. The anteroposterior 
braking impulse in downhill running was almost double that of the propulsive impulse. In level running, these two impulses were not significantly different. The peak impact vertical force during downhill running at $-12 \%$ gradient is approximately $1800 \mathrm{~N}$ or 2.8 times body weight ${ }^{34}$.

\section{Anterior compartment}

Friden and colleagues have concentrated on the effects of eccentric muscle action on the anterior compartment of the leg 22,51. This compartment has non-elastic fascial sheaths and may be predisposed to anterior tibial compartment syndrome with repeated downhill running.

Eccentric exercise causes a significant increase in intramuscular pressure in a non-compliant compartment, compared with concentric exercise. Muscle fibre swelling is a predominant feature after eccentric exercise. This was thought to be a factor in the development of DOMS in a tight compartment ${ }^{51}$. However, intramuscular pressure is raised immediately after eccentric exercise, before the muscles become painful. Once pain starts there are still the same raised pressures. Newham and Jones' work $^{52}$ on DOMS in the biceps, found there was no detectable difference in pressure between sore and control arms. It appears therefore that eccentric exercise may cause an increase in pressure in non-compliant compartments (with the possibility of compartment syndrome) but is not directly associated with DOMS pain ${ }^{51}$.

It has been reported ${ }^{53}$ that anterior compartment syndrome develops mainly in runners, while soccer players and cyclists develop posterior compartment syndrome. Perhaps there is a connection between the eccentric work done in the anterior compartment in runners, to control forefoot loading. This movement is not found in cyclists. In downhill running there may be more eccentric work done by these muscles, which may contribute to a higher incidence of compartment syndrome.

\section{Summary}

There are striking differences in the timing of events in stance phase between downhill and level running, although contact phase is independant of downhill angle $^{34}$. Peak knee flexion angle, peak flexion velocity, maximum ankle dorsiflexion and peak dorsiflexion velocity all occur significantly later in stance phase in downhill running ${ }^{45}$. Possibly because there is a longer period of negative work for the knee extensors and ankle flexors, there is more resultant muscle damage.

Variation in intensity of the eccentric exercise may be the reason for the differences in both time course and magnitude of PCK response between downhill running and high intensity eccentric exercise. Most protocols using high-intensity eccentric exercise were careful to eliminate all concentric components from the tests. In contrast, downhill running is a much more functional activity, combining concentric and eccentric exercise. The physiological consequences of downhill running seem to be closer to normal reactions to muscle damage with moderate PCK increases. There is evidence to suggest that a prior bout of eccentric exercise does reduce decrements in strength, severity of muscle damage and perceived muscle soreness after a subsequent bout of downhill running.

\section{References}

1 Newham DJ, McPhail G, Mills KR, Edwards RHT. Ultrastructural changes after concentric and eccentric contractions in human muscle. J Neurol Sci 1983; 61: 109-22.

2 Armstrong RB, Ogilvie RW, Schwane JA. Eccentric exerciseinduced injury to rat skeletal muscle. J Appl Physiol 1983; 54: 90-3.

3 Friden J, Sjostrom M, Ekblom B. A morphological study of delayed onset muscle soreness. Experientia 1981; 237: 506-7.

4 Friden J, Seger J, Ekblom B. Sublethal muscle fibre injuries after high-tension anaerobic exercise. Eur J Appl Physiol 1988; 57: 360-68.

5 Friden J. Changes in human skeletal muscle induced by long term eccentric exercise. Cell Tissue Research 1984; 236: 365-72

6 Armstrong RB. Muscle damage and endurance events. Sports Med 1986; 3: 370-81.

7 Appell HJ, Soares JM, Duarte JA. Exercise, muscle damage and fatigue. Sports Med 1992; 13: 108-15.

8 Armstrong RB, Warren GL, Warren JA. Mechanisms of exercise induced muscle fibre injury. Sports Med 1991; 12: 184-207.

9 Clarkson PM, Nosaka K, Braun B. Muscle function after exercise-induced muscle damage and rapid adaptation. Med Sci Sports Exerc 1992; 24: 512-20.

10 Clarkson PM, Tremblay I. Exercise-induced muscle damage, repair and adaptation in humans. J Appl Physiol 1988; 65: 1-6.

11 Sargeant AJ, Dolan P. Human muscle function following prolonged eccentric exercise. Eur J Appl Physiol 1987; 56: 704-11.

12 Eston RG, Critchley N, Baltzopoulos V. Delayed-onset muscle soreness, strength loss characteristics and creatine kinase activity following uphill and downhill running. J Sports Sci 1994; 12: 135.

13 Fitts RH, Courtwright JB, Kim DH, Witzmann FA. Muscle fatigue with prolonged exercise: contractile and biochemical alterations. Am J Physiol 1982; 242: C65-73.

14 Byrd SK, McCutcheon LJ, Hodgson DR, Gollnick PD. Altered sarcoplasmic reticulum function after high intensity exercise. J Appl Physiol 1989; 67: 2072-77.

15 Jones DA, Round JM. Skeletal Muscle in Health and Disease: A textbook of muscle physiology. Manchester, UK: Manchester University Press. 1990; 158-74.

16 Finney S, Eston RG, Baltzopoulos V. Muscle soreness and strength loss changes after downhill running following a prior bout of isokinetic eccentric exercise. J Sports Sci 1994; (in press).

17 Newham DJ, Jones DA, Ghosh G, Aurora P. Muscle fatigue and pain after eccentric contractions at long and short length. Clinical Science 1988; T4: 553-7.

18 Jones DA, Newham DJ, Torgan C. Mechanical influences on long-standing human muscle fatigue and delayed-onset pain. J Physiol 1989; 412: 415-27.

19 Cleak MJ, Eston RG. Muscle soreness, swelling, stiffness and strength loss after intense eccentric exercise. $\mathrm{Br}$ J Sports Med 1992; 26: 267-72.

20 Edwards RHT, Mills KR, Newham DJ. Measurement of severity and distribution of experimental muscle tenderness. J Physiol 1981; 317: 1-2P.

21 Newham DJ, Mills KR, Quigley BM, Edwards RHT. Pain and fatigue after concentric and eccentric muscle contractions. Clin Sci 1983; 64: 55-62.

22 Fridén J, Sfakianos PN, Hargens AR. Blood indices of muscle injury associated with eccentric muscle contractions. J Orthop Res 1989; 7: 142-45.

23 Jones DA, Newham DJ, Round JM, Tolfree SE. Experimental human muscle damage: morphological changes in relation to other indices of damage. J Physiol. 1986; 375: 435-48.

24 Newham DJ, Jones DA, Edwards RHT. Plasma creatine kinase changes after eccentric and concentric contractions. Muscle-Nerve 1986; 9: 59-63. 
25 Evans WJ, Meredith CN, Cannon JG, Dinarello CA, Frontera WR, Hughes VA, Jones BH, Knuttgen HG. Metabolic changes following eccentric exercise in trained and untrained men. $J$ Appl Physiol 1986; 61: 1864-68.

26 Newham DJ, Jones DA, Clarkson PM. Repeated high-force eccentric exercise: effects on muscle pain and damage. J Appl Physiol 1987; 63: 1381-86.

27 Costill DL, Pascoe DD, Fink WJ, Robergs RA, Barr SI, Pearson D. Impaired muscle glycogen resynthesis after eccentric exercise. J Appl Physiol 1990; 69: 46-50.

28 Byrnes WC, Clarkson PM, White JS, Hsieh SS, Frykman PN, Maughan RJ. Delayed onset muscle soreness after repeated bouts of downhill running. J Appl Physiol 1985; 59: 710-15.

29 Schwane JA, Williams JS, Sloan JH. Effects of training on delayed muscle soreness and serum creatine kinase activity after running. Med Sci Sports Exerc 1987; 19: 584-90.

30 Schwane JA, Johnson SR, Vandenakker CB, Armstrong RB. Delayed-onset muscle soreness and plasma CPK and LDH activities after downhill running. Med Sci Sports Exerc 1983; 15: 51-6.

31 Dick RW, Cavanagh PR. An explanation of the upward drift in oxygen uptake during prolonged sub-maximal downhill running. Med Sci Sports Exerc 1987, 19: 310-17.

32 Pierrynowski MR, Tudus PM, Plyley MJ. Effects of downhill or uphill training prior to the downhill run. Eur J Appl Physiol 1987; 56: 668-72.

33 Westerlind KC, Byrnes WC, Mazzeo RS. A comparison of the oxygen drift in downhill vs. level running. J Appl Physiol 1992; 72: 796-800.

34 Iverson JR, McMahon TA. Running on an incline. J Biomech Eng 1992; 114: 435-41.

35 Komi PV, Buskirk ER. Effect of eccentric and concentric muscle conditioning on tension and electrical activity of human muscle. Ergonomics 1972; 15: 417-34.

36 Schwane JA, Armstrong RB. Effect of training on skeletal muscle injury from downhill running in rats. J Appl Physiol 1983; 55: 969-75.

37 Friden J, Seger J, Sjostrom M, Ekblom B. Adaptive response in human skeletal muscle subjected to prolonged eccentric training. Int J Sports Med 1983; 4: 177-83.

38 Jones DA, Newham DJ. The effect of training on human muscle pain and muscle damage. J Physiol. 1985; 365: 76P.
39 Byrnes WC, Clarkson PM. Delayed onset muscle soreness and training. Clin Sports Med 1986; 5: 605-14.

40 Clarkson PM, Byrnes WC, Gillison E, Harper E. Adaptation to exercise-induced muscle damage. Clin Sci 1987; 73: 383-86.

41 Miller G, Wilcox A, Schwenkel J. The protective effect of a prior bout of downhill running on delayed onset muscle soreness (DOMS). Med Sci Sports Exerc 1988; 20: S75.

42 Sforzo GA, Lamb DR. Muscle soreness after exercise: effects of early training with concentric contraction. In: Dotson CO, Humphrey JH, eds. Exercise Physiology: current selected research. New York, USA: AMS Press, 1985; 171-79.

43 Ebbeling CB, Clarkson PM. Muscle adaptation prior to recovery following eccentric exercise. Euro J Appl Physiol 1990; 60: 26-31.

44 Walmsley B, Hudgson JA, Burke RE. Forces produced by medial gastrocnemius and soleus muscles during locomotion in freely moving cats. J Neurophysiol 1978; 41: 1203-16.

45 Buczec FL, Cavanagh PR. Stance phase knee and ankle kinematics and kinetics during level and downhill running. Med Sci Sports Exerc 1990; 22: 669-77.

46 Milliron MJ, Cavanagh PR. Saggital plane kinematics of the lower extremity during distance running. In: Cavanagh PR (ed) Biomechanics of Distance Running, Champaign, Il, USA: Human Kinetics Publishers, 1990.

47 Leiber RL, Fridén J. Muscle damage is not a function of muscle force but active strain. J Appl Physiol 1993; 74: 520-26.

48 Root ML, Orien WP, Weed JH. Normal and Abnormal Functions of the Foot. Clinical Biomechanics Vol 2. Los Angeles, USA: Clinical Biomechanics Corporation, 1977.

49 Winter DA. Moments of force and mechanical power in jogging. J Biomech 1983; 16: 91-7.

50 Dick RW, Cavanagh PR. A comparison of ground reaction forces (GRF) during level and downhill running at similar speeds. Med Sci Sports Exerc 1987; 19: S12.

51 Fridén J, Sfakianos PN, Hargens AR. Muscle soreness and intramuscular fluid pressure: comparison between eccentric and concentric load. J Appl Physiol 1986; 61: 2175-79.

52 Newham DJ, Jones DA. Intra-muscular pressure in the painful human biceps. Clin Sci 1985; 69: (Suppl): 27.

53 Styf J. Chronic exercise-induced pain in the anterior aspect of the lower leg. An overview of diagnosis. Sports Med 1989; 7: 331-39. 